\title{
The Nature of the Computing and Natural Science in Engineering Education
}

\author{
${ }^{1}$ Bojadzievski Andonova and ${ }^{2}$ Ramesh kulkarni \\ ${ }^{1,2}$ School of Computer Science and Information Technology, \\ ${ }^{1,2}$ University American College Skopje, Skopje 1000, North Macedonia \\ ${ }^{1}$ Andonovavesi3221@outlookmail.com
}

\begin{abstract}
Article Info
Journal of Computing and Natural Science (http://anapub.co.ke/journals/jens/jens.html)

Doi: https://doi.org/10.53759/181X/JCNS202101011

Received 15 December 2020; Revised form 18 January 2021; Accepted 22 March 2021.

Available online 05 July 2021.

○2021 Published by AnaPub Publications.
\end{abstract}

\begin{abstract}
In engineering, the interdisciplinary essence of the Computing and Natural Science (CNS) as well as its relations with other fields are described. This paper presents a discussion of the phases by which CNS education evolve from the recognition of initial growth in the '80's to current growth. The limitations and potential benefits of varying CNS education methodologies are addressed, and so is the advancement of the number of the foundational elements, which are common to most strategies. The CNS course content, grades and curriculum are examined and all bachelors' programs are surveyed. The curricula of the various programs are examined and discussed for their relative weighting for the standard "toolkit."

Keywords - Computing and Natural Science (CNS), Science, Technology, Engineering and Mathematics (STEM)

\section{INTRODUCTION}

The last decade has witnessed significant progress in engineering and science, which was partly driven by massive improvements in networking and telecommunication power and prevalence. These developments have been taken into account through the development of new techniques for digital technology that allow us to better comprehend technologies with exponential growth and authenticity. Each example includes econometric analysis, primary particular quark structure, manufacturing processes, process technology, drug discovery, astrophysics, non-linear interplay and turbulent behaviour, biological diversity, financial services and undermining of large data sets such as virtual human beings and electronic sky monitoring centers.
\end{abstract}

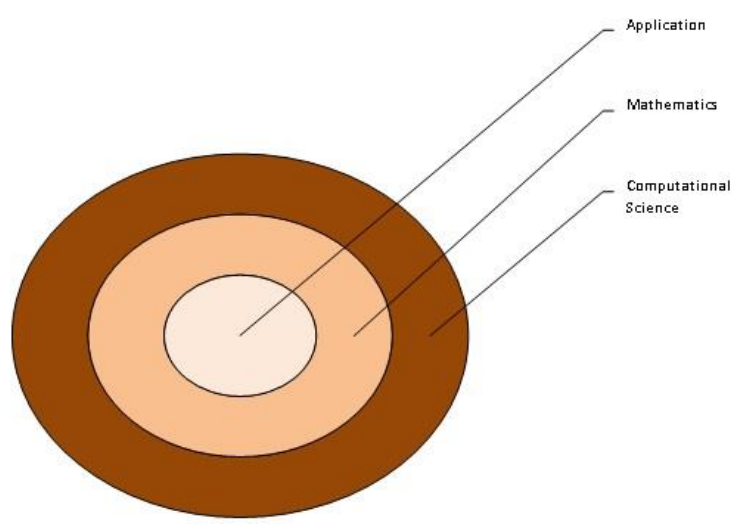

Fig 1. Computing and Natural Science (CNS) in the field of Engineering as an interdisciplinary activity linking computation with science and mathematics.

"Computing and Natural Science (CNS)" is a concept that is defined differently in Engineering. It sometimes refers to the interdisciplinary conjunction of computer applications, technologies and knowledge that are required to address real world problems in engineering and science [1]. In other cases, it refers to engineering and science which employed computational models or sometimes indicates that computer expertise and the materials necessary for application are being researched and developed. The initial CNS display is presented on the left side of Fig 1 and signifies an overlap between computational mathematics, informatics and systems. In addition to the aforementioned concerns of other fields of study, a new, central circle reveals that the CNS includes its key components, which unite and connect other disciplines. This earliest outlook is now substituted by the one over the right of Fig 1.

The CNS core can be regarded as a computer tool and method collection and a troubleshooting mindset using the insights from one field of study to resolve issues in another [2]. This CNS core is now included in Science, 
Technology and Mathematics (STM) classes, journals and curriculums at different educational levels, combining research problems resolution and computing. Section I has introduced the concept of CNS and presented the definition of it. Section II below presents an analysis of the relevant literature related to the concept. Section III presents a critical analysis of the concept in engineering education. Section IV presents the results while Section V concludes the paper.

\section{LITERATURE REVIEW}

Z. Zhou, C. Tang, Y. Liu, Y. Liu and J. Xu in [3] argue that computer design and simulation and visual analytics appear to benefit most scientific disciplines. Indeed, in addition to experimental research and theory, the newly-found and broad-based significance of computing brought about a paradigm shift of science investigation to include natural science methodologies. Computation and visual analytics enable us to generate understanding into major concerns which are too intricate or difficult to mathematically study, or too costly to study experimentally. For instance, fuel concentration, ignition energy, and heat wave computation can be studied in a combustion chamber over 3000 Kelvin, gluon flow tube configuration between proton quarks and potential earth-asteroid collision orbits.

G. Dong, P. Sun, W. Shi and C. Choi in [4] argue that the valuation of the CNS to the scientific community increases with more scholars incorporating simulation into their work. CNS workgroups in particular allow other researchers to continue pursuing their scientific research interests without incurring additional costs trying to develop codes and algorithms. These task forces have been based on the identification, research and improvement of computing methodologies and software components prevalent to several applications as well as on the experimentation of the software's performance on different hardware. The application of statistical databases on discrete mathematics, specific functionalities and additional mathematical models has been one feature of the CNS project teams. The main computer calculations involve these sub-processes that are highlighted in numerous CNS educational programmes.

S. Wang and J. Liu in [5] agree that the CNS society is concerned to ensure that these libraries are accurate, efficient, robust, portable and scalable as they are transferred to various powerful computational configurations. These achievements have helped advance with mainframes and are now being used more extensively with the stupendous increase in computer power. CNS also supports and implements simulation technology that is widely applicable to computational subroutine libraries. Consider for instance the "particle composition" computer technology in which each particular particle is formed and its journey is accompanied in time by the suitable energy equation.

According to Z. Yang and Z. Ge in [6], this procedure is employed to simulate systems as diversified as the installation of sediments in one tank, the conception of planets and stars, gas and fluid particles, the development of nanostructures, the interplay of motor particles, and ash nanoparticle movement in advanced manufacturing burners. Many fields of science evidently benefit from improving the methodology or teaching its professionals to effectively use it. CNS technologies and their affiliated libraries are increasing in size, representing an inestimable prosperity of tools and resources. In the CNS society they are already heavily dependent and we anticipate that dependence to increase in the future. That's why we need CNS education to transmit this affluence to scientific and engineering generation to generation in the future.

In last decade, the PITAC Council has acknowledged Information and communication technology as one of their driving forces for growth in the economy [7]. The Committee identifies the need to provide information and digital technology for about one million people, something that can't only be fulfilled by all IT departments of the organisation fully. The Science \& technology Committee reports reiterated the issues of the community and business laboratories that their rapidly expanding needs for informational and computer technology skilled professionals cannot be met.

Moreover, federal project activities like DOE-ASCI and the Presidential IT Initiatives are based on scientists and computer programmers. The exponential increase in computer power in the past decade and a dramatic reduction in computer costs are the consequence of those needs for more people with computer skills. Few aspects of life have changed in so short a period of time by 1000 variables. Children are currently playing games on class computers that were perceived supercomputers 10 years ago and only accessible in government laboratories. As several areas of daily life, at home and at work are now permeated by computer systems, they also fundamentally affect the market for technological work. One way is to place individuals who need knowledge in the field outside of their schooling and (obsolete) career paths. Having multiple qualifications and substantial qualifications is therefore considered as a way to improve commercialization and sustainability of work.

Z. Ramji and J. Etowa in [8] argue that employers seem to be favorably inclined towards people who have received education in several professions, as they can recruit fewer and keep them longer. However, it is time consuming and expensive to attain advanced degrees, as is the case in one directorate and in several other degrees. By comparison, a learning interdisciplinary CNS scheme saves time and money for learners who otherwise would be able to attend multiple courses. It delivers great, consistent and lowest possible duplication education and empowers learners with a multidisciplinary toolkit (which will be defined soon) that has proved to have been useful in a variety of subject areas. 


\section{CRITICAL ANALYSIS}

\section{The CNS Education Nature}

Science education and engineering, partially triggered by federal government reports and subsidies, has reacted to developments in computer science. In 1989, the Technology and Science Regulation Office questioned the system of education to: 1) raise the percentage of learners projected for career opportunities in fields such as Science, Technology, Engineering and Mathematics (STEM) and 2) enhance mathematics, science and computer science of all learners. The National Science Foundation recently stated and promoted computing as an adequate pedagogy for mathematics and science.

The NSF report by Wright and Chorin prompted even high schoolers to set up computational mathematics training (CNS's rudimentary strategy). Traditional science principles tend to be concentrated in hypothesis. CNS learning, in juxtaposition, provides computer knowledge of the sciences of computer simulations. It tries to teach scientific knowledge using the investigation method whereby the computer functions is a virtual workshop simulating nature. Evidence is provided as required rather than as artifacts for memorization needed to facilitate the survey.

The CNS view provides standardized mathematics and science education as a result of its problem-solving disposition. This also constitutes a substantial number of engineering and technology theories easier for students to access, for instance learners who are motivated to learn about hardware, software and algorithms for their sake. Moreover, CNS enhances its scientific curriculum through widening the plethora of issues available for study to include issues with which it can have no analytical solutions.

CNS-supported learning types have been explored by the National Foundation for Educational Improvement. These include a student-centered or constructivistic perspective that indicates that when students interact effectively with, rather than only receive, knowledge, they start learning. The CNS methodology often focuses on the research work and on the team, as well as on knowledge construction. When this combination of elements succeeds, nonengaged, risky learners can be transformed into engaged and committed students. A CNS education, that is, general to specific, is well suited to quantitative learning. Students begin with the understanding about the limited variety of underlying scientific legislation governing nature and systems, with additional detail and computational analyzes as required. When the arithmetic ability of the learners is limited, they can be comprehended through use of images without examining scientific and mathematical specifics. Compared to the conventional students' perceptions of scientific knowledge as intricate, this supports the view that natural events are basically simple. It also provides a fundamental foundation for students to build upon as their skills grow. This capability to stimulate students' curiosity as well as provide comprehensive learning through intriguing and rational illustrations is a primary motivation in which educators apply master latest technology.

\section{Phases of the CNS Education}

CNS education has evolved through different steps. Initially, engineers and scientists were able to learn or receive from their consultants' hand-down computational modeling they considered necessary to solve issues. The first structured phase, estimated (1980-1990), the concept of recognition. In it, professionals identified something unique and not necessarily the best. The 1982 Lax study, which recognized the fundamental change in calculations as fundamental strategies of scientific knowledge, was epitomized for this phase. In order to make the transition easier, the report recommends that funders provide more training in scientific computing.

CNS education's second phase, ca. 1990-2000, was the "childhood". The ideas of the CNS began being instructed by people who knew those concepts through investigation in some new or existing courses. Phase 3 , approx. ca 2000 to 2010 was considered for "Early Growth." It is now the phase we're taking and is categorized both at the undergraduate level and at graduate level by a range of courses and curricula. Although we presume the maturity stage of CNS learning to emulate what we have presently, in the midst of a dramatic change, it is difficult to make reliable forecasts. However, one might anticipate the continuation of the numbers of CNS courses and programs either by deliberately introducing computing, or implicitly by recruiting professors with specializations in scientific knowledge, innovative technologies, mathematics and engineering.

In Computational X, $\mathrm{X}$ has become a defined subject such as algebra, biology, accounting, cosmology, science or sociology, and we are foreseeing an increasing variety of courses. The pressure might originate from multiple sources for the different courses. The Industrial areas and National laboratories have progressively shown that scientists and philosophers are not satisfied with their needs of computation. Moreover, there are undergraduates intrigued in using their newly-found skills in discipline $\mathrm{X}$ in established CNS programmes. In the X departments, there are educators who want their learners to master the skill of calculating or revitalizing their classes. Finally, there are the CNS professionals (including the scholars) who assume that computer education is necessary to serve the public in proposition.

A computer X program may be able to make starting a computer Y program that shares certain of the same classes easier. Following the establishment of a number of computer programs in any school, they can decide for the sake of effectiveness and cooperation to be organized under the CNS module. These modules can operate within Discipline X as multidisciplinary programs or as independent CNS departments. Such autonomous departments might have a basis department of mathematics, computer engineering and other associated disciplines. The latest CNS units could also be driven by funders, who start to promote the CNS more explicitly. In addition, internally driven universities and colleges implement the progression to redefine into multidisciplinary units, emphasizing social problem-solving 
competency. Their affiliation and their scientific orientation to resolve issues will be beneficial to these units. A selfsufficient CNS unit could also offer the advantages of other departments to a CNS minor. A CNS minor should include more than the customary science or engineering student's core knowledge of computing approaches and methodologies, but not an entire range of subjects projected of a CNS major.

\section{Demands for CNS Education}

CNS is a field driven by research which links computing-interested faculty to those who are interested in applications, because calculations must be included in modern science problem solving (Major disciplines in Fig 1). Both CNS and computer science have a proficiencies with an interest in computer hardware and software, a faculty which is interested in numerical processes and found both in CNS and applied mathematics; and both in CNS and application proficiency which is interested in a computerized solution for extending experimental and theoretical work.

The Faculty tends to be attuned by CNS as it offers a way to integrate its tools and advancements in contemporary courses. This can also save time by taking students to a dimension where they can assist educators in the process of analysing data. Contrary to that, learners learn and acquire beneficial professional skills through the tools and technologies required by contemporary practitioners. While incorporating your analytics tools into courses that you teach is ideal, it has to be acknowledged that both students and faculty have a massive burden of educational, investigations and regulatory requirements of a CNS program. Since new instruments continuously come to scientific and industrial workplaces, in a sector that educates information technologies use, we cannot preserve a statistical education system. Similarly, it is also challenging especially that new programs are developed.

CNS courses often have high-end research facilities, workspaces, quantum computers or the Beowulf structures in addition to curriculum design. It can be an important task to maintain this equipment with always-aged software and hardware. Although a committed system manager can be very important, as can the resources of a microprocessor centre, a faculty member depends on the location, strategic planning and expenditure for a computer laboratory. The students' (quite legitimate) expectancy that faculty members will be aware of all CNS courses, including those taught by professionals in other departments, raises additional requirements for CNS professors. Concerns about recruitment and promotion judgements arise where Faculty 1) has joint meetings in a variety of departments; 2) has additional requests or 3 ) is engaged in research activities which are hard to evaluate by traditional discipline.

A CNS learner can face more requirements, as is possible for all interdisciplinary education, than a learner with only single specialty. CNS learners need lessons, information and guidance in several areas to begin with. Moreover, students can feel the added stress of mastering three subjects and mastering just one of their peer groups in a single discipline. Moreover, pressure often comes into play in the need to deal with an education system that is unproven and changing rapidly, particularly when no department can call "residence." However, multidisciplinary learning can also create new and enriching "realms" for learners. If both the sciences and the computer are their academic interests, then they are free to take up those aspirations. This should endear to learners who are willing, as well as to those learners who recognize that this is a contemporary solution to tackling the challenges of various majors and degree courses.

\section{CNS Education and its Intellectual Content}

Whereas there is a fundamental recognition that Computational sciences are not similar to Computer Science, at the moment, there is not locally accredited curriculum for CNS. The first CNS courses to be taught in most schools were identified based on their growing dependencies on sophisticated tools for research computation. Most of these courses represented adjustments to the regular curriculum that is provided by national centers of supercomputers and projected to focus on training machines instead of focussing on basic education. Other programs were provided within the department as a major segment of the graduate-leaner research program and some amounted to degree that integrated computing (considered as a suffix) i.e. the Michigan framework. Most of the Undergraduate programs were soon considered, and currently there are categorized as K-12 with CNS content.

In particular, a the undergraduate levels, most of the understanding of more intellectual CNS [11-12] content and education is required to appreciate the various methodologies applied to education. There are however a number of undergraduate programs in the field of Computational Science to make an assumption about. Basically, the particular subjects required to provide this sophisticated content is still a prevailing concern in various researchers. Consequent to that, the definition to follow is retrieved from the survey results and experience in formulating two different degree programs. In this research paper (according to the data obtained from the American Institute of Physics, students have five years of study and their educational aspect is most fundamental in the present employment market.

We visualize that for graduates whose major employment field is STEM skills, which remain to be significantly fundamental for the graduates that seek job opportunities in software development and computer programming. The significance of computer skills and mathematics is also evaluated in report released by the National Science Board [9]. According to the report, approximately $74 \%$ of computer-science and mathematics doctorates work in the same department according to their qualification contrary to about $51 \%$ of individuals with degrees in life science and physical science. This similar trend has been seen at the bachelor's level with estimations of $35 \%$ against $22 \%$.

The results indicate that the demands of problem-solving, computer and mathematics skills in the job market, including the significance of science education, are fundamental in multiple jobs. It is considerable that the incorporation of computer and mathematics skills with engineering and science education is a critical investment, mostly for undergraduate (who are more likely to find an employment opportunity outside their major) than graduate 
students. Determining the best balance of science computing and mathematics is a key concern that we will evaluate soon.

The assessment of the learning outcomes for learners is fundamental for the introduction of the CNS courses. This is mostly the case for CNS where it might be easier to expect an individual course to train learners everything they have to know concerning a particular subject. Historically, student learning outcomes have been governed by the needs of the research, which is, in the top-down manner. We only know of a single work that has been published and recommend a collection of student learning outcomes for various levels of the CNS learning. Based on the perspectives of learners, a normal student learning outcomes for a CNS learning system integrates:

- Learning top-notch performance computing and computing languages

- Accepting knowledge from applied mathematics and computing approaches

- Learning the foundation of modelling and simulation

- Mastering how to analyse and interpret data in a visual manner both after an during computation

- Applying the essential computing competency to about a single area of application

- Mastering how to communicate remedial approaches and results.

The above meaning learning outcomes for students are accomplished by students mastering various operating and subjective computational issues. Due to the fact that course in different departments might significantly cover similar course materials, instead of a list of courses, we provide the 6 general knowledge areas, which are required to attain the student learning outcomes (see Fig 2)

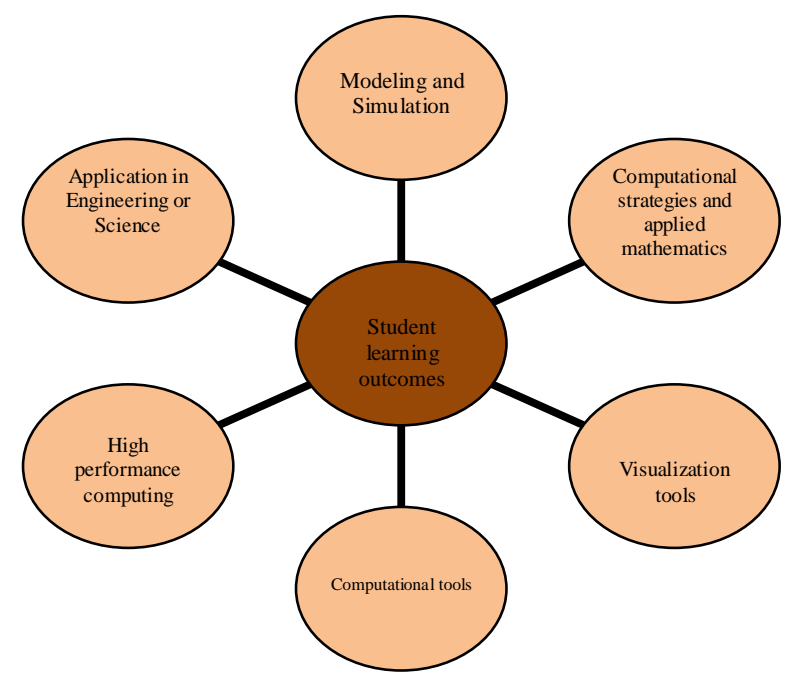

Fig 2. The general areas of knowledge for the student learning outcomes

Even though not all the same weight, the components are all essential and have to be tackled in different dedicated programs and their dimensions.

\section{Tools for Computation}

Despite the fact that computing itself is a fundamental tool for CNS, it is critical to provide environments and situations in which students are confortable while using computers. Typically, the programs in which this happens are normally known as "Fundamentals In" or "Introduction To;" Computer Science, Computational Tools, Problem Solving, Modeling, or Computational Science. Much knowledge can be accomplished through various courses e.g. Data Structures, Advance Software Tools, and Numerical Linear Algebra. Student learning outcomes integrate:

- $\quad$ Programming in more languages that are compiled e.g. Java, C++, C, or Fortran90;

- Capacity to operate using Windows and Unix operating systems;

- Familiarity with issue-solving ecosystems e.g. Math-Ematica, Macsyma, Matlab, Maple for both symbolic and numeric computations;

- Familiarity with floating-points numerical and computations e.g. Monte Carlo approaches, Partial differential equation, Differentiation, and Integration;

- Application of mathematical subroutine repositories and libraries e.g. Jama, NetSolve, ScaLaPack, and Blas;

- Application of two-dimensional and 3D software packages for visual representation such as AVS, Xmgr, Gnuplot and VisAD.

\section{High-Performance Computing}

Skills for high-performance computing hardware and software are vital when programming computer-intensive application. The various subjects incorporate high-level languages, optimizing methodologies, and integrated parallel 
computing. The related theoretical skills might come from different course in the theoretical framework of Programming languages and Computational Architecture. The student learning outcomes integrate:

- Programing on PC clusters or supercomputers;

- Comprehending the program precision, performance benchmarks, memory hierarchy, and program speed;

- Comprehending the connection among performance, language, and architecture;

- Experience with company's benchmarks e.g. the Linpack benchmark;

- Familiarity with most of the parallel libraries e.g. MPJ, MPI and PVM

\section{Computational Strategies and Applied Mathematics}

Basic knowledge in this case derives from different courses in Vector Calculus, Differential Integral, certainly with basis on various examples and applications. Differential equations and Numerical analysis might provide more specific knowledge, as courses such as Computational Physics, Physical Sciences, Computational Methods, and Applied and Computational Mathematics.

Lastly, at least a single course in Statistics and Probability is required whereby; real-life lab data is apt. The learning outcomes incorporate:

- The skillset of the computational approaches for numerical solutions of differential and integral equations, such as adaptive mesh, mesh generations, discrete ordinates, discrete particles, finite element, finite differences and high-order solver for ODE e.g. Monte-Carlo, Fast Fourier Transforms, Runge Kutta approaches.

- Familiarity with more particular differential equation in engineering and science

- Application of the scientific subroutine libraries

- Matrix computation with scientific routine library.

\section{Modeling and Simulation}

This subject is fundamental to accomplish the CNS objectives in solving real-life problems. The competencies might be addressed in multiple courses e.g. modelling and simulations, Computational X, issue-solving and vibrant system specific learning outcomes incorporate:

- Being familiar with the exact and appropriate motion equations for different natural systems. Samples integrate the continuity of most equations, protein folding, population dynamics, energy, and equation for momentum;

- Familiarity with the various phases (Issue, Theory, Framework, Implementation, and Evaluation) in modelling is applicable to diversified fields. Samples integrate finance, dynamical systems, climate modelling, quantum mechanics, drug design, genetics, molecular dynamics, electromagnetics, and engine combustion;

- Capacity to undertake statistically comprehensive evaluation of data from both simulations and experiments

\section{Visualization Tools}

Being familiar with the visualization software for post-simulation and run-time data is fundamental. Tools should have the capacity to handle big data, allow two-dimensional and three-dimensional plots, including dicing and slicing for high-dimensional data and generate figures for publication. The dimensions have to be that of OpenGL, Gnuplot, AAceGr, Matlab and AVS. The programs administered for these skills integrate Computational tools, Visualization tools and scientific computation.

\section{Engineering and Science Application}

A fundamental segment of CNS learning is having learners to concentrate on more traditional fields of interest e.g. engineering, art, criminal justice, business, earth science, biology, physics and chemistry. This should assist the learners in the employment market to apply in the graduate school, and in comprehending realistic and different approaches of focussing on these problems. Just which segment and programs can help attain this objective effectively is an element of local student interest, expertise, and local politics. Typically, a computational X program is fundamental for this aim since it gives the required exposure to the applications while presenting students to a number of computational aspects of the $\mathrm{X}$ disciplines.

\section{RESULTS}

To formulate a comprehensive quantitative understanding of the intellectual content and the CNS courses, we have evaluated the curricula for CNS programs that we are cognizant. Our vital purpose is to provide the required materials for informed and critical discussions concerning the status and nature of CNS training. The choices of the programs were stimulated by the data retrieved from conferences, Web portal for computational sciences at Suny, including the Swanson survey regarding CNS courses. Regarding the survey for graduate degree courses, the Swanson's survey was preferably used, including the SIAM working group on CNS courses. Swanson is capable of identifying a single 
B.S. course in Computational Sciences, 3 courses in Computational Physics and various minors and concentrations. According to the SIAM research, a single Brockport as the CNS program was identified. We integrated all the Swanson's program in our evaluation other than the University of Chicago where we did not find any samples. Even though it did not show up in internet searches, we incorporate a degree program at the UC Berkeley based on the existence that is linked to personal use. The analysis in this paper is detailed in its coverage in Physical representatives, computational degrees and computer science degree.

According to the Swanson survey, it is possible to identify where course are in existence and in providing enough details regarding the contents of various computational programs (the same as the initial descriptions of the standardized toolkit [10]. The evaluation is to follow is considerably general. For every course, we evaluate the 4 years' valuation of programs and count the various courses, which can be grouped into Applications, Mathematics and Computing among others. It has to be admitted that our evaluation is crude. We assess neither the materials incorporated in the selective programs, nor the others, which might include applications, mathematics or computing. However fascinating it might be to visualize how nations integrate their CNS courses, we have fundamentally restricted our analysis in America. This is an effect of out challenges in evaluating and determining how to count the classes and requirements for global systems, and retrieving updated data from the various webpages. We know that the top-notch quality CNS courses in colleges e.g. the University of Waterloo, University of Singapoor, Trimity College in Dublin and Kanazawa University in Japan for connection to both international and national CNS program.

Fig 4 below shows mean percentage of the overall curriculum integrated to Application, Mathematics and computing, including "other" for B.S. program in (from right to left) i.e. Physics, Computational Physics, Computational Engineering \& Science, and Computer Science. The mean for the computer science is from the Oregon State University, University of Illinois Champaign Urbana and Carnegie Mellon University; CNS mean is based on the Berkeley University and the Suny Brockport; CP mean in the Illinois State University, Oregon University and Suny Buffalo. The Physics mean is from the Oregon University and Champaign Urbana University in Illinois.

\begin{tabular}{ccccc}
\hline \multicolumn{5}{c}{ Balance of subjects and courses } \\
\hline $\mathbf{0}$ & $\begin{array}{c}\text { Computational } \\
\text { Sciences }\end{array}$ & $\begin{array}{c}\text { Computational } \\
\text { Sciences and } \\
\text { Engineering }\end{array}$ & $\begin{array}{c}\text { Computational } \\
\text { Programming }\end{array}$ & Physics \\
Comp & 40.0 & 20.0 & 20.0 & 2.0 \\
Math & 12.0 & 23.0 & 19.0 & 17.0 \\
App & 17.0 & 28.0 & 28.0 & 45.0 \\
Other & 31.0 & 29.0 & 12.0 & 36.0 \\
\hline
\end{tabular}

Fig 3. Mean percentage of the general curriculum integrated to Application, Mathematics and computing, including "other" for B.S. program in (from right to left) i.e. Physics, Computational Physics, Computational Engineering \& Science, and Computer Science.

Generally, we might say that the column to the left in Fig 4 indicates a firm computing but weaker application element in degrees for Computer Science programs; the column to the right indicates a stronger application but a weaker element in Physics degrees. The two different versions of the computing courses shown in Fig 4 are visualized to provide uniform and the same balance among multiple elements. Contrary to our evaluation, 35\% of the students' career are projected to be the majors in the fields of Engineering or Science, approximately $25 \%$ in Science or Mathematics (aside from the major field), about $25 \%$ in arts and humanities, and about $15 \%$ in the electives. Our assessment indicates that the application-centred computational training is more firm in the field of Mathematics due to its computational contents.

\section{CONCLUSION}

CNS learning concentrates on typical "toolkit" of subjects, which have been regarded as fundamental tools used to mitigate real-life challenges. Whereas most of these subjects might cover the programs addressed by the traditional faculties, we have drawn conclusions for differential CNS programs, which integrate all the tools, develop effective problem-solving perspectives, glue the interdisciplinary programs inclusively, and develop a sense of self-worth in the computational society. Course offerings and local politics are considered effective to determine the manner in which colleges select their means of integrating CNS in Engineering within its programs. In that case, we make assumptions that CNS contents of undergraduate training will further diversify, either through the incorporation of CNS into contemporary programs, by providing certain Computational $\mathrm{X}$ courses or by issuing the Computational $\mathrm{X}$ degrees.

Indubitably, the most effective approach to provide well-established and coherent training is by a special graduation program at CNS. It is also most likely to further the sector and ensure continuity in the institution. As colleges formulate various Computational X courses, combining their programs into the CNS faculty can be more efficient and easier. These units can be used by a minor in CNS to serve even more departments. It seems, however, that a computational X degree offers the same learning experience. There seem to be significant connections and cooperation between the individuals who lead the new CNS programs and those who want to launch such programs. In fact, the NSF is supporting efforts. We are now sharing course material and providing the required guide on how to develop a more stable curriculum in newly developed courses. The advancement of learning outcomes for the students, an agreement on the standardized CNS 
ISSN: 2789-181X

Journal of Computing and Natural Science 1(3)(2021)

program and the formulation of top-notch quality books will assist our graduates to develop their fields and place themselves.

\section{References}

[1]. W. Yang, K. Li and K. Li, "A hybrid computing method of SpMV on CPU-GPU heterogeneous computing systems", Journal of Parallel and Distributed Computing, vol. 104, pp. 49-60, 2017. Available: 10.1016/j.jpdc.2016.12.023.

[2]. S. Pllana and J. Barhen, "Introduction to the computing special issue: performance portability and tuning for multi-core and many-core computing systems", Computing, vol. 96, no. 12, pp. 1113-1114, 2013. Available: 10.1007/s00607-013-0379-0.

[3]. Z. Zhou, C. Tang, Y. Liu, Y. Liu and J. Xu, "Visual Analytics for Multidimensional Time-Varying Data via Dimension Reduced Visual Perception", Journal of Computer-Aided Design \& Computer Graphics, vol. 30, no. 7, p. 1194, 2018. Available: 10.3724/sp.j.1089.2018.16713.

[4]. A. Haldorai and A. Ramu, "Security and channel noise management in cognitive radio networks," Computers \& Electrical Engineering, vol. 87, p. 106784, Oct. 2020. doi:10.1016/j.compeleceng.2020.106784

[5]. A. Haldorai and A. Ramu, "Canonical Correlation Analysis Based Hyper Basis Feedforward Neural Network Classification for Urban Sustainability," Neural Processing Letters, Aug. 2020. doi:10.1007/s11063-020-10327-3

[6]. G. Dong, P. Sun, W. Shi and C. Choi, "A novel valuation pruning optimization fuzzing test model based on mutation tree for industrial control systems", Applied Soft Computing, vol. 70, pp. 896-902, 2018. Available: 10.1016/j.asoc.2018.02.036.

[7]. S. Wang and J. Liu, "Community robustness and its enhancement in interdependent networks", Applied Soft Computing, vol. 77, pp. 665-677, 2019. Available: 10.1016/j.asoc.2019.01.045.

[8]. Z. Yang and Z. Ge, "Monitoring and prediction of big process data with deep latent variable models and parallel computing", Journal of Process Control, vol. 92, pp. 19-34, 2020. Available: 10.1016/j.jprocont.2020.05.010.

[9]. P. Purwoko, "Auditing Information System : Delivery Product Service", CommIT (Communication and Information Technology) Journal, vol. 5, no. 1, p. 21, 2011. Available: $10.21512 /$ commit.v5i1.554.

[10]. Z. Ramji and J. Etowa, "Workplace Integration: Key Considerations for Internationally Educated Nurses and Employers", Administrative Sciences, vol. 8, no. 1, p. 2, 2018. Available: 10.3390/admsci8010002.

[11]. U. Ghani, X. Zhai and R. Ahmad, "Mathematics skills and STEM multidisciplinary literacy: Role of learning capacity", STEM Education, vol. 1, no. 2, p. 104, 2021. Available: 10.3934/steme.2021008

[12]. K. Arnone and D. Hanuscin, "An Exploratory Cross-Sectional Survey Study of Elementary Teachers' Conceptions and Methods of STEM Integration", Journal of Research in STEM Education, vol. 4, no. 2, pp. 159-178, 2018. Available: 10.51355/jstem.2018.43. 